\title{
Seguimiento médico de los niños con síndrome de Down
}

\author{
Health supervision for children with Down syndrome
}

\author{
Karla Adney Flores-Arizmendi, ${ }^{1}$ Tania Tonantzin Vargas-Robledo, ${ }^{2}$ Gabriela Eguiarte-Díaz, ${ }^{3}$ Lidia del Carmen \\ Gómez-Puente ${ }^{4}$
}

\section{ANTECEDENTES}

La identificación de la alteración en el cromosoma 21 ha permitido catalogar las principales comorbilidades coexistentes en las personas con síndrome de Down. Desde 1866 John Langdon Down realizó una descripción de algunos problemas médicos en las personas con trisomía 21, pero no fue sino hasta 1970 cuando Coleman efectuó una revisión de las comorbilidades y estudios de laboratorio y gabinete que regularmente deben practicarse en esta población. ${ }^{1}$ En 1990 Pueschel y colaboradores publicaron las primeras Guías de Atención en síndrome de Down. ${ }^{2}$ A raíz de esto, numerosos grupos interesados en el síndrome de Down han desarrollado, en diversos países y regiones, programas de salud y recomendaciones de seguimiento médico. ${ }^{3,4,5}$ Hasta la fecha no existen guías de práctica clínica con estricto rigor metodológico; sin embargo, cada día se llevan a cabo numerosas investigaciones, lo que ha contribuido de manera decisiva al incremento de la expectativa de vida y la mejora en la salud y, por ende, de la calidad de vida de las personas con síndrome de Down. Por esto fue que se efectuó esta revisión, a fin de dar a conocer las pautas que deben seguirse en la valoración médica de las personas con síndrome de Down conforme a su grupo de edad.

\section{Seguimiento en atención primaria}

La atención a pacientes con diversos tipos de discapacidad representa un reto en la práctica médica habitual. Es un escenario al que nos enfrentamos sin haber recibido, en muchas ocasiones, la enseñanza correspondiente en nuestra formación profesional. Tener el conocimiento básico de enfermedades frecuentes, como el síndrome de Down, es fundamental para brindar la atención completa y de calidad que merecen todos los pacientes.

En México, la Norma Oficial Mexicana que revisó en forma amplia la atención integral de las personas con discapacidad, se publicó en

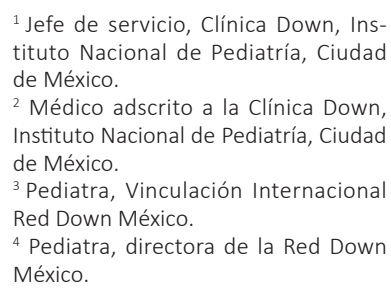

${ }^{2}$ Médico adscrito a la Clínica Down, Instituto Nacional de Pediatría, Ciudad de México.

${ }^{3}$ Pediatra, Vinculación Internacional Red Down México.

${ }^{4}$ Pediatra, directora de la Red Down México.

Recibido: 8 de octubre de 2020

Aceptado: 19 de marzo de 2021

Correspondencia

Karla Adney Flores-Arizmendi

adneyss@gmail.com

Este artículo debe citarse como: FloresArizmendi KA, Vargas-Robledo TT, Eguiarte-Díaz G, Gómez-Puente LC. Seguimiento médico de los niños con síndrome de Down. Acta Pediatr Méx 2021; 42 (3): 142-8. 
el Diario Oficial de la Federación en 1998; la cual se actualizó en 2012. ${ }^{6}$ Además, en 2007, la Secretaría de Salud publicó un lineamiento técnico: "Atención integral de la persona con síndrome de Down".?

En el ámbito mundial se estima una incidencia de este síndrome en 1 por cada 600 a 700 recién nacidos. La Organización Mundial de la Salud estima una prevalencia en 1:1000 y 1:1100 nacidos vivos. ${ }^{8}$ En México, el Registro y Vigilancia Epidemiológica de Malformaciones Congénitas (RYVEMCE) informó una prevalencia de 11.37 casos de síndrome de Down por cada 10,000 nacimientos, para el periodo 2004-2008. ${ }^{9}$

\section{Control de salud por edades}

\section{Visita prenatal}

La consulta prenatal da la oportunidad de asesorar a una familia en la que se ha establecido el diagnóstico, in útero, de síndrome de Down. Si bien en México el diagnóstico prenatal sigue siendo poco frecuente $(4.7 \%$ de acuerdo con la Guía de Práctica Clínica del año 2011) ${ }^{10}$ es importante considerar los siguientes puntos:

1. Estudios prenatales por ultrasonido y métodos diagnósticos invasivos y no invasivos efectuados que llevaron al diagnóstico.

2. Asesoría genética prenatal para explicar los posibles mecanismos de la alteración genética y riesgo potencial de recurrencia. ${ }^{10}$

3. Manifestaciones clínicas más frecuentes. ${ }^{11}$

4. Estudios de laboratorio y gabinete que ayuden a identificar enfermedades asociadas con el síndrome de Down.

5. Tratamientos e intervenciones disponibles en la actualidad.

6. Importancia de incluir los aspectos positivos de las personas con síndrome de Down (expectativa de vida, logros, inclusión y oportunidades).

\section{Neonatal (del nacimiento al primer mes)}

El primer paso en toda evaluación de un recién nacido en el que se sospeche síndrome de Down es una exploración física minuciosa, apoyada en los criterios de Hall, ${ }^{3}$ y la integración de una historia clínica con antecedentes prenatales, incluidos los estudios diagnósticos. De ser posible, el diagnóstico debe comunicarse a ambos padres, posterior a la recuperación de la madre, en presencia del recién nacido. Se incia con la felicitación por el nacimiento de su hijo; esto no siempre se hace $y$, ante todo, no debiera olvidarse que se trata del nacimiento de un ser humano. ${ }^{12}$ La forma en cómo se da esta primera noticia marcará, de forma trascendental, el futuro del niño y la familia. La medicina humanista y el acompañamiento empático facilitarán esta etapa de duelo por la que cursan los padres.

El seguimiento durante esta etapa es el siguiente:

1. Cariotipo, asesoría y consejería por Genética.

2. Evaluación por un cardiólogo pediatra que incluya ecocardiograma (incluso si no hay soplos o manifestaciones clínicas). ${ }^{13}$

3. Pruebas de función tiroidea completas o revisión del tamiz metabólico (T4 y TSH) para descartar hipotiroidismo congénito. ${ }^{14}$

4. Practicar el tamiz auditivo al nacimiento (emisiones otoacústicas). Complementar con potenciales evocados cuando el reporte del tamiz sea desfavorable en más de dos ocasiones (antes de los 3 meses). ${ }^{15}$

5. Valoración por el oftalmólogo pediatra, con tamiz oftalmológico, en los primeros tres meses de vida, para descartar: catarata congénita, nistagmo, estrabismo y dacrioestenosis. ${ }^{16}$ 
6. Si hay vómito o ausencia de heces: descartar malformación gastrointestinal (atresia duodenal, malformación anorrectal, enfermedad de Hirschsprung). ${ }^{17}$

7. Vigilar la alimentación para asegurar que la ingesta calórica sea la adecuada. Insistir en la importancia de la alimentación al seno materno (técnica adecuada, problemas de succión y deglución, frenillo corto, paladar hendido o submucoso, etc.).

8. Biometría hemática para descartar algún trastorno mieloproliferativo neonatal transitorio. $^{18}$

9. Iniciar un programa de intervención temprana que incluya terapia física y orofacial, acorde con el estado de salud de cada niño.

10. Referir a un grupo de padres para fomentar redes de apoyo. Atender las preocupaciones de los padres. ${ }^{19}$

\section{Infancia (del primer mes al primer año)}

Todo niño en este periodo debe tener un seguimiento pediátrico regular de crecimiento y desarrollo. El control del crecimiento deberá registrarse en gráficas especiales para población con síndrome de Down, con vigilancia de la relación peso-talla. ${ }^{20,21}$ El desarrollo psicomotor deberá evaluarse con sus correspondientes intervalos, con base en los hitos del desarrollo para población con síndrome de Down. ${ }^{22} \mathrm{El}$ seguimiento deberá ser el siguiente:

1. Evaluación pediátrica de niño sano para vigilar la adecuada ganancia de peso y talla.

2. Evaluación por el cardiólogo pediatra en caso de no haberse efectuado al nacimiento. Considerar la hipertensión pulmonar, incluso en ausencia de cardiopatía.
3. Pruebas de función tiroidea completas a los 6 y 12 meses.

4. Pruebas de audición. Seguimiento con emisiones otoacústicas, timpanometría y potenciales evocados auditivos a los 6 meses de edad.

5. Examinar los oídos, la nariz y la garganta. Los niños con estenosis de conductos auditivos externos, en quienes no se logren visualizar las membranas timpánicas con visión directa en el otoscopio, deberán referirse a la atención del otorrinolaringólogo.

6. Examen de la agudeza visual, sobre todo si hay nistagmo, estrabismo o sospecha de baja visión (a los seis meses y, posteriormente, cada año).

7. Inicio de la alimentación complementaria entre los 4 y 6 meses de vida, de acuerdo con las recomendaciones de las guías más recientes de la ESPGHAN y la Academia Americana de Pediatría. ${ }^{23}$ Deben tomarse en cuenta el peso y los datos de madurez motora: adecuado sostén cefálico y disminución del reflejo de extrusión; y ofrecer alimentos ricos en hierro.

8. Biometría hemática a los 6 y 12 meses. Vigilar trastornos hematológicos (anemia macrocítica, linfopenia, trombocitopenia, síndrome mieloproliferativo neonatal transitorio). Dar respuesta a las dudas de los padres.

9. Aconsejar el ingreso a programas de intervención temprana que incluyan terapia física, orofacial y ocupacional. Dar respuesta a las dudas de los padres.

10. Esquema de inmunizaciones completo. Aplicar la vacuna de influenza estacional anual y las no incluidas en la Cartilla 
Nacional de Vacunación (primeras dosis de varicela, hepatitis A y meningococo) ${ }^{24}$

\section{Niñez y adolescencia (del primer año a los 18 años)}

1. Continuar con la consulta pediátrica del niño sano, utilizando las tablas de peso y talla para niños con síndrome de Down. Llevar el seguimiento del índice de masa corporal en niños mayores de 2 años y del índice cintura-talla. Vigilar que no se Ilegue al sobrepeso u obesidad mediante la orientación de buenos hábitos de alimentación y actividad física.

2. Ecocardiograma y valoración cardiológica según lo indicado por el cardiólogo o cada dos años en ausencia de cardiopatía.

3. Pruebas de función tiroidea cada año.

4. Valoración audiológica anual.

5. Examen anual de la vista. Los defectos de refracción son muy comunes y pueden producir ambliopía entre los 3 y 5 años, al igual que el estrabismo no tratado. En adolescentes debe descartarse el queratocono.

6. Comentar con los padres sobre los signos y síntomas de apnea obstructiva del sueño. Respiración oral, ronquidos, sueño intranquilo, pausas respiratorias durante el sueño, posiciones extrañas para dormir, despertares frecuentes, somnolencia diurna, trastornos de conducta. ${ }^{25}$ Valorar la conveniencia de la interconsulta con el otorrinolaringólogo.

7. Examen bucodental semestral, a partir de la erupción del primer diente, para corrección de la dentición primaria y secundaria. Aplicar la profilaxis para endocarditis bacteriana, en caso de caries dental.
8. Descartar la enfermedad celiaca con base en los síntomas sugerentes (diarrea o estreñimiento, retraso en el crecimiento, anemia, dolor abdominal y distensión crónica, alteraciones conductuales). Valorar con estudios de anticuerpos anti-transglutaminasa tisular, IgA total y anticuerpos antiendomisio. ${ }^{26}$

9. Indagar si ha tenido síntomas de inestabilidad atlanto-axial (dolor de cuello, debilidad, cambios en el tono muscular, alteraciones en la marcha o en la función de esfínteres y síntomas de compresión medular.) Solicitar una radiografía lateral de la columna cervical, en caso de querer practicar deportes de alto impacto. En caso de cualquier cirugía, comentar esta condición con el cirujano y anestesiólogo para consideraciones de manipulación de la vía aérea (cuidados del cuello por riesgo de inestabilidad atlantoaxoidea). ${ }^{27}$

10. Esquema de inmunizaciones completo. Complementar el esquema nacional con vacunas adicionales: neumococo 23 valente, segunda dosis de varicela y de hepatitis A y refuerzo de meningococo.

11. Seguimiento por un terapeuta del lenguaje para asegurar el máximo desarrollo de la comunicación verbal.

12. Continuar con el programa para el desarrollo motor e intelectual.

13. Descartar trastornos conductuales, déficit de atención, autismo, y otros. Consultas psicopedagógicas periódicas para valorar avances y controlar los conflictos y problemas de conducta. ${ }^{28}$

14. En las adolescentes practicar la evaluación ginecológica correspondiente. 
15. Iniciar charlas de temas de educación sexual y prevención de abuso. Indicar métodos anticonceptivos si es necesario. ${ }^{19}$

16. Orientar sobre métodos de enseñanza y adaptaciones curriculares de acuerdo con sus habilidades. Evaluaciones psicopedagógicas para individualizar la educación según las aptitudes.

17. Iniciar la transición y seguimiento a la vida independiente a partir de los 16 años. Considerar cuestiones legales de tutoría y elaboración de plan financiero a largo plazo. ${ }^{29}$

En el Cuadro 1 se resume el seguimiento médico por edades.

Dentro del espectro de comorbilidades que pueden llegar a manifestar las personas con síndrome de Down, hay que considerar las descritas en el Cuadro 2, de acuerdo con los síntomas para referirlos con el especialista que corresponda.

\section{CONCLUSIONES}

Estas recomendaciones pueden utilizarse como apoyo y referencia a los profesionales de la salud para brindar una atención eficiente; el reconocimiento temprano de enfermedades mejora el pronóstico y calidad de vida y disminuye el costo de atención de las complicaciones para los sistemas de salud.

Consideramos decisivo llevar a cabo más estudios y profundizar en el conocimiento que permitan elaborar guías de práctica clínica con estricto rigor metodológico que den pie al avance en la atención médica y la implementación de programas prioritarios, dirigidos a mejorar la calidad de vida de esta población.

Desde la última actualización de las recomendaciones en salud de 2011 de la Academia Americana de Pediatría se han adquirido nuevos conocimientos. En el año 2020 se publicó un artículo de revisión de comorbilidades en las personas con síndrome de Down, donde se abor-

Cuadro 1. Seguimiento médico de niños y adolescentes con síndrome de Down

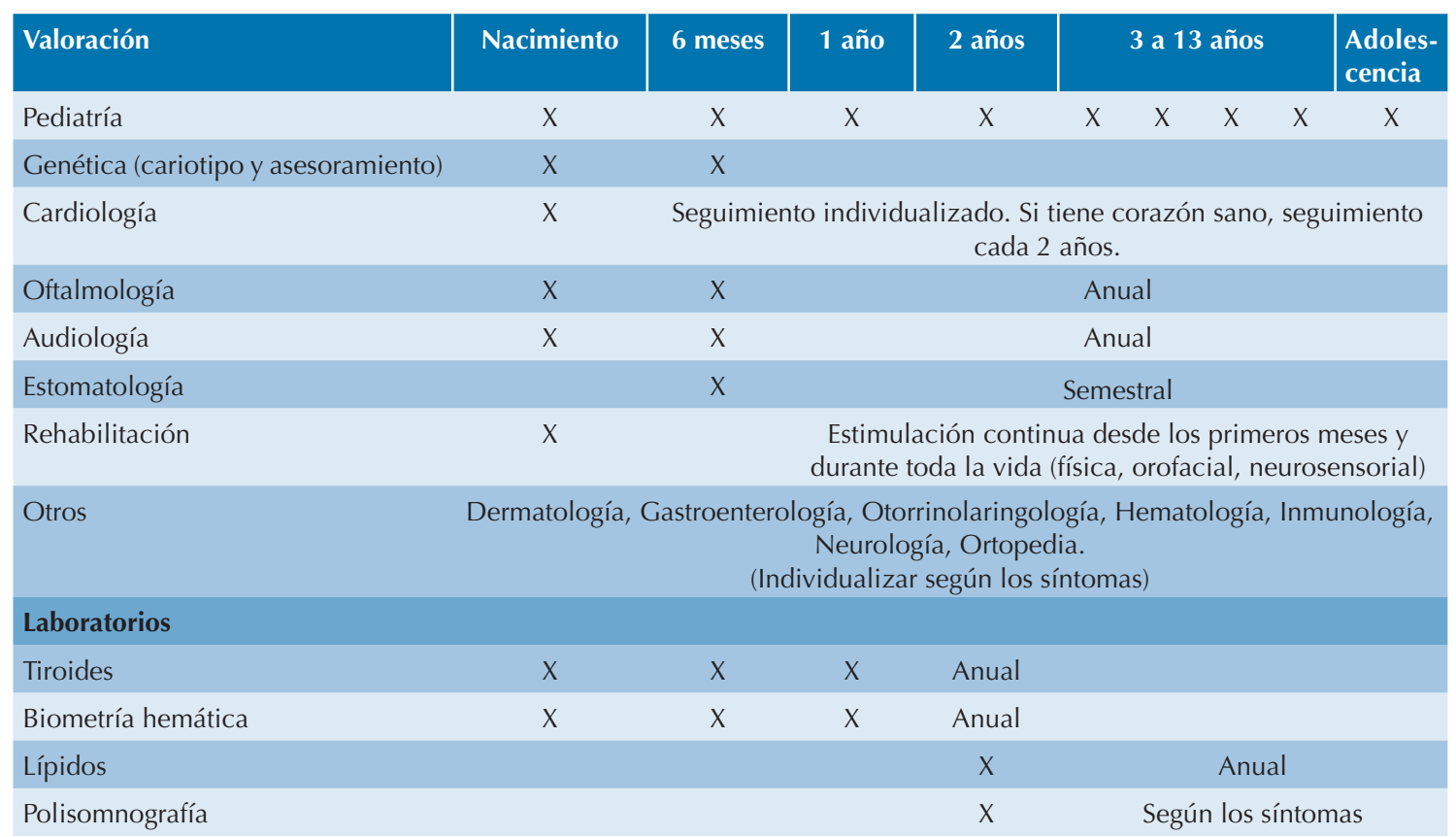


Flores-Arizmendi KA, et al. Seguimiento médico de los niños con síndrome de Down

Cuadro 2. Seguimiento individualizado según las comorbilidades

\begin{tabular}{l|l}
\hline Especialidad & \multicolumn{1}{c}{ Enfermedad a descartar } \\
\hline Gastroenterología & Reflujo, estreñimiento crónico, trastornos de mal absorción, enfermedad celiaca. \\
\hline $\begin{array}{l}\text { Dermatología } \\
\text { Otorrinolaringología }\end{array}$ & Xerosis, vitíligo, alopecia, dermatitis seborreica, onicomicosis. \\
\hline Hematología & Anemia, neutropenia, linfopenia, síndrome mieloproliferativo transitorio del recién nacido, leucemia. \\
\hline Inmunología & Deficiencia selectiva de anticuerpos, inmunodeficiencias primarias. \\
\hline Neurología & Epilepsia, autismo, trastornos de atención. \\
\hline Ortopedia & Displasia del desarrollo de cadera, pie plano, escoliosis, hallux valgus, inestabilidad atlanto-axial. \\
\hline
\end{tabular}

dan temas de importancia, como la medicina integrativa y la transición a la vida adulta. ${ }^{30}$ Con el fin de complementar estas recomendaciones, el equipo de la Clínica de Atención del Niño con Síndrome de Down del Instituto Nacional de Pediatría está trabajando en una revisión narrativa de los temas que no han sido incluidos o que requieren actualización, entre ellos: dislipidemia, hiperbilirrubinemia en el recién nacido, disfunción tiroidea, deglución, inmunidad, vacunas, hipertensión pulmonar, diabetes mellitus, hiperuricemia, micronutrientes y autismo. Esperamos su publicación en breve.

\section{REFERENCIAS}

1. Diefendorf AO, Bull MJ, Casey-Harvey D, Miyamoto RT, Pope M. Down syndrome: a multidisciplinary perspective. J Am Acad Audiol 1995; 6 (1): 39-46.

2. Pueschel SM. Clinical aspects of Down-syndrome from infancy to adulthood. Am J Med Genet 1990; 56 (7): 52-56. https://doi.org/10.1002/ajmg.1320370708.

3. Hanson JW, Lloyd-Puryear M, Moore CA, Williams J, Hoyme $\mathrm{HE}$, Bull MJ. Health supervision for children with Down syndrome. Pediatrics 1994;107 (2): 442-449. doi: 10.1542/ peds.107.2.442.

4. Lizama MC, Retamales NM, Mellado CS. Recomendaciones de cuidados en salud de personas con síndrome de Down: 0 a 18 años. Rev Med Chil 2013; 141 (1): 80-89. doi:10.4067/ S0034-98872013000100011.

5. Borrel J, Flórez J. Programa peninsular de salud para personas con Síndrome de Down. Fed Iberoam Sindr Down 2015; 1 (12): 13-47.

6. Flores K, Gomez L del C, Boissoneautl D. Manual y Guia de Atención Medica a personas con síndrome de Down. Acad Mex Pediatría 2016; 1: 54-76.
7. Secretaría de Salud. Atención integral de las persona con síndrome de Down. 1a ed. México: Centro Nacional de Equidad de Género y Salud Reproductiva, 2007.

8. National Down Syndrome Society. What Is Down Syndrome? - National Down Syndrome Society. National Down Syndrome Society. doi:10.1007/s10557-010-6249-5.

9. Sierra Romero M del C, Hernández EN, Serrano SC, Pablo AER, Hernández JV. Prevalencia del síndrome de Down en México utilizando los certificados de nacimiento vivo y de muerte fetal durante el periodo 2008-2011. Bol Med Hosp Infant Mex 2014; 71 (5): 292-97. doi:10.1016/j. bmhimx.2014.09.002

10. Secretaría de Salud. Diagnóstico prenatal del síndrome de Down. Guía Pract Clin. 2011.

11. Bull MJ. Health supervision for children with down Syndrome. Pediatrics. 2011; 128 (2): 393-406. doi:10.1542/ peds.2011-1605

12. Carroll C, Goloff N, Pitt N. When Bad News Isn' t Necessarily Bad: Recognizing Provider Bias When Sharing Unexpected News. Pediatrics 2018; 142 (1): e20180503. https://doi. org/10.1542/peds.2018-0503.

13. Versacci P, Di Carlo D, Digilio MC, Marino B. Cardiovascular disease in Down syndrome. Curr Opin Pediatr 2018; 30 (5): 616-22. doi:10.1097/MOP.0000000000000661.

14. Kariyawasam D, Carré A, Luton D, Polak M. Down syndrome and nonautoimmune hypothyroidisms in neonates and infants. Horm Res Paediatr 2015; 83 (2): 126-31. doi:10.1159/000370004.

15. Chin CJ, Khami MM, Husein M. A general review of the otolaryngologic manifestations of Down Syndrome. Int J Pediatr Otorhinolaryngol 2014; 78 (6): 899-904. https:// doi.org/10.1016/j.ijporl.2014.03.012.

16. Afifi $H H$, Azeem AAA, El-Bassyouni HT, Gheith ME, Rizk $A$, Bateman JB. Distinct ocular expression in infants and children with down syndrome in cairo. Egypt myopia and heart disease. JAMA Ophthalmol 2013; 131 (8): 1057-66. doi:10.1001/jamaophthalmol.2013.644.

17. Bermudez B, De Oliveira C, De Lima M, Neiva M, Celli A. Gastrointestinal disorders in Down syndrome. Am J 
Med Genet Part A 2019; 179 (8): 1426-31. doi:10.1002/ ajmg.a.61258

18. Sarbay H. Evaluation of hematological findings in children with Down syndrome. Int J Contemp Pediatr 2018; 5 (5): 1724. doi:10.18203/2349-3291.ijcp20183506.

19. Virji-Babul N, Eichmann A, Kisly D, Down J, Haslam RHA. Use of health care guidelines in patients with Down syndrome by family physicians across Canada. Paediatr Child Health (Oxford) 2007; 12 (3): 179-83. doi:10.1093/pch/12.3.179.

20. Zemel B, Pipan M, Stallings V, Hall W, Schadt K. Growth charts for children with Down syndrome in the United States. Pediatrics 2015; 136 (5): 1204-1211. doi:10.1542/peds.2015-1652.

21. Durán XP, Domech LQ, Corretger $\mathrm{M}$, Calzada $\mathrm{D}$, Subirachs RG, Santamaría AS. Tablas de crecimiento actualizadas de los niños españoles con síndrome de Down. Pediatrika 2004; 8: 34-46.

22. Winders P, Wolter-Warmerdam K, Hickey F. A schedule of gross motor development for children with Down syndrome. J Intellect Disabil Res 2019; 63 (4): 346-56. https://doi. org/10.1111/jir.12580.

23. Fewtrell M, Bronsky J, Campoy C. Complementary feeding: A position paper by the European Society for Paediatric Gastroenterology, Hepatology, and Nutrition (ESPGHAN) committee on nutrition. J Pediatr Gastroenterol Nutr 2017; 64 (1): 119-32. doi:10.1097/MPG.0000000000001454.
24. Corretger Rauet JM. Vacunaciones en el niño con síndrome de Down. Pediatr Aten Primaria 2014; 123 (4): 475-81. doi:10.4321/S1139-76322014000300010

25. Maris $M$, Verhulst $S$, Wojciechowski $M$, Van de Heyning $P$, Boudewyns A. Prevalence of obstructive sleep apnea in children with Down syndrome. Sleep 2016; 39 (3): 699-704. doi:10.5665/sleep.5554.

26. Mazurek D, Wyka J. Down syndrome genetic and nutritional aspects of accompanying disorders. Rocz Państwowego Zakładu Hig 2015; 66 (3): 189-94.

27. El-Khouri M, Mourão MA, Tobo A, Battistella LR, Herrero CFP, Riberto M. Prevalence of atlanto-occipital and atlantoaxial instability in adults with Down Syndrome. World Neurosurg 2014; 132 (6): 1121-27. doi:10.1016/j. wneu.2014.02.006.

28. Oran $\mathrm{M}$, Oxelgren $\mathrm{U}$, Cathrine $\mathrm{E}$, Holmbom A, Isaksson A. Prevalence of autism and attention-deficit -hyperactivity disorder in Down syndrome: a population-based study. Dev Med Child Neurol 2016; 59 (3): 276-83. doi:10.1111/ dmcn.13217.

29. Diamandopoulos K, Green J. Down syndrome: An integrative review. J Neonatal Nurs 2018; 34 (2): 165-72. doi:10.1016/j.jnn.2018.01.001.

30. Bull MJ. Down syndrome. N Engl J Med 2020; 382 (24): 2344-52. doi:10.1056/NEJMra1706537. 